\title{
The Role of Laparoscopic Total Colectomy in Treatment of Slow- Transit Constipation: A Prospective Study
}

\author{
Mohamed Shaaban Khalifa, MD; Mohamed Ibrahim Hasan, MD; \\ Ahmed Hussein Abdel hafez, MD.
}

Department of Surgery, Ain Shams University, Cairo, Egypt.

Background: Patients with intractable chronic constipation should be evaluated with physiologic tests after structural disorders, and extracolonic causes have been excluded. Conservative treatment options should be tried excessively. If surgery is indicated, subtotal or total colectomy with ileorectal anastomosis is the treatment method of choice.

Patients and methods: In this prospective study, 7 patients were reviewed including patient demography, clinical manifestations, preoperative investigations, operative procedure, intraoperative and postoperative complications, and postoperative follow up. All patients enrolled in the study underwent laparoscopic total colectomy (LTC), with ileorectal anastomosis

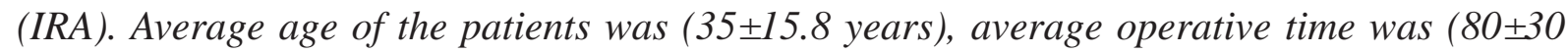
$\mathrm{min})$, average blood loss was ( $75 \pm 30 \mathrm{ml})$, average hospital stay was (6 \pm 2.5 days).

Results: 7 patients in our study were diagnosed to have slow-transit constipation via history, clinical examination, colonoscopy and barium enema to rule out structural disorders, colonic motility study, anorectal manometry, colonic transit time, and defecography. All patients underwent LTC with IRA after failure of conservative treatment. 3 patients(42.8\%) had postoperative diarrhea which resolved spontaneously after 2 weeks, 1 patient (14.2\%) had wound infection, 1patient(14.2\%) had minor anastomotic leak which subsided on conservative treatment. 2 patients had abdominal pain and bloating which improved gradually, 1 patient had recurrent constipation which was improved on laxatives, median number of motions/day was 3 , with median satisfaction and success rate of about $82 \%$.

Conclusion: Laparoscopic treatment of slow-transit constipation seems to be safe, feasible, and effective procedure with satisfactory results, with minimal postoperative complications, short hospital stay, with a faster return to social activity, along with a better cosmetic results and good functional results.

Key words: Slow-transit constipation, laparoscopic total colectomy(LTC), ileorectal anastomosis(IRA), colonic motility study, anorectal manometry.

\section{Introduction:}

Constipation is one of the most frequent gastrointestinal symptoms, and one of the most frequent reasons for medical consultation. Basically constipation is related to intestinal motility disorders, pelvic floor disturbance. ${ }^{1}$ Patients often complaint of subjective feelings like incomplete evacuation, abdominal or rectal pain, firm stool consistency, and need for straining. The best definition for constipation was proposed by Whitehead. ${ }^{2}$ Two or more of the following complaints must be present when the patient is not taking laxatives, and if symptoms persist for at least 12 months:

1) Straining on $>25 \%$ of bowel movements.

2) Feeling of incomplete evacuation after $>25 \%$ of bowel movements.

3) Scyballous stools on $>25 \%$ of bowel movements.

4) Stools less frequent than $2 /$ week with or without other symptoms.

Slow-transit constipation occurs most commonly in young women who had 
infrequent bowel movement, the condition often starts at puberty, associated symptoms are an infrequent urge to defecate, bloating, and abdominal pain, dietary and cultural factors contribute to symptoms, in these patients a high fiber dietary, increase stool-weight, decrease colon transit time. ${ }^{3}$ Patients with more severe slow-transit constipation have a poor response to dietary fiber and laxatives, such patients have a more delayed emptying of the proximal colon, and fewer high-amplitude peristaltic contractions. Colonic inertia, a related condition is characterized by slow colonic transit. Histopathological studies have shown alterations in the number of the mynteric plexus neurons expressing the excitatory neurotransmitter substance $\mathrm{P}$, abnormalities in the inhibitory neurotransmitters vasoactive intestinal peptide and nitric oxide. Hirschsprung's disease is an extreme form of slow-transit constipation with similar enteric neuropathological features. ${ }^{4}$

A thorough history and physical examination can rule out most secondary causes of constipation. An assessment of stool form can be used to estimate the extremes of colonic transit time. A careful rectal examination should be performed, the perianal area should be examined for scars, fistulas, fissures, external piles. Next the clinician should observe the perineum with the patient at rest and while the patient is bearing down, in order to determine the extent of perineal descent, digital rectal examination should determine whether fecal impaction, anal stricture, or rectal masses are present or not. Laboratory tests that may be useful include thyroid-function tests, serum calcium, glucose, and electrolytes, a complete blood count, and urinalysis. 5,6

A complete colon examination is required to exclude structural diseases such as colon cancer, which is done via colonoscopy and barium enema. Physiological testing is necessary in patients with refractory symptoms which include anorectal manometry and balloon expulsion, defecography may be considered if the results of these tests are equivocal. The colonic transit time is normally less than 72 hours. Other diagnostic tests include electromyography, and pudendal nerve terminal motor latency. 7,8

Management regimens include; high fluid intake, physical exercise, increased fiber intake, osmotic laxatives such as milk of magnesia, sorbitol, lactulose, colonic stimulants like bisacodyl, senna derivatives. Prokinetic agents should be preserved for patients with severe constipation. Patients with defecatory disorders should undergo retraining of the evacuation process. Patients with fecal impaction should have manual evacuation, preliminary data suggest that injection of botulinum type A toxin into the puborectalis muscle may be effective in treatment of defecatory disorders. ${ }^{9,10}$

For refractory constipation, total colonic resection and ileorectal anastomosis should be considered only if the patient does not have a defecatory disorders and only after medical therapy have failed. ${ }^{11}$

\section{Patients and methods:}

In this prospective study, 7 patients were evaluated, had full investigations, and underwent laparoscopic total colectomy and ileorectal anastomosis, between May 2013 and May 2015, and the study was conducted at January 2016. Patients had full preoperative diagnostic work up in the form of history taking, physical examination, colonoscopy and barium enema, CT scan, MRI, anorectal manometry, colonic motility studies, and colonic transit time estimation, to assess slowtransit constipation. All patients tried medical treatments with no or poor response, as well as defecatory disorders were absent in all patients. All the patients were fully informed about the procedure, and written consent was taken. A complete patient demographic data, preoperative investigations, and operative data are illustrated in Table (1).

Operative technique: After a mechanical bowel preparation. The patient is placed in a modified lithotomy position, and pneumoperitoneum is established. Using the subumbilical 12-mm trocar the camera is inserted. Two further $12-\mathrm{mm}$ and $5-\mathrm{mm}$ trocars, each are used for access to the 


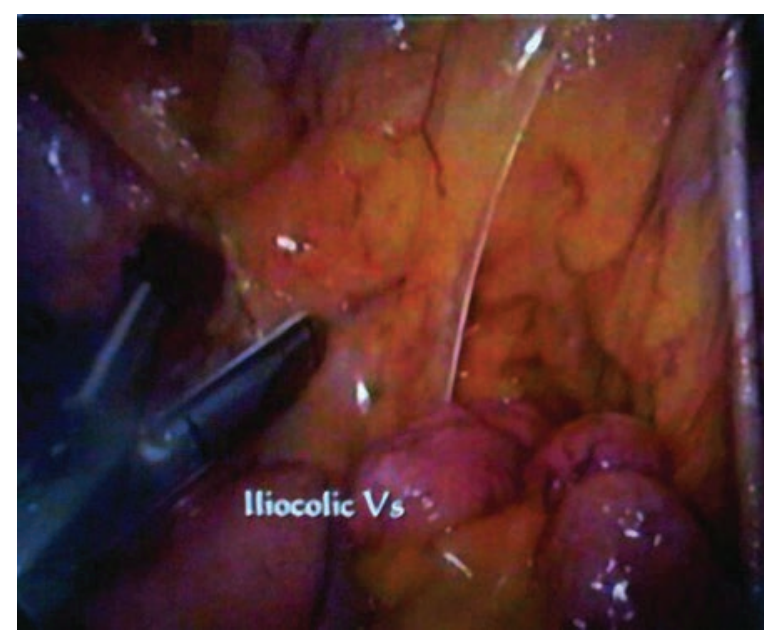

Figure (1): Division of iliocolic vessels, ileum.

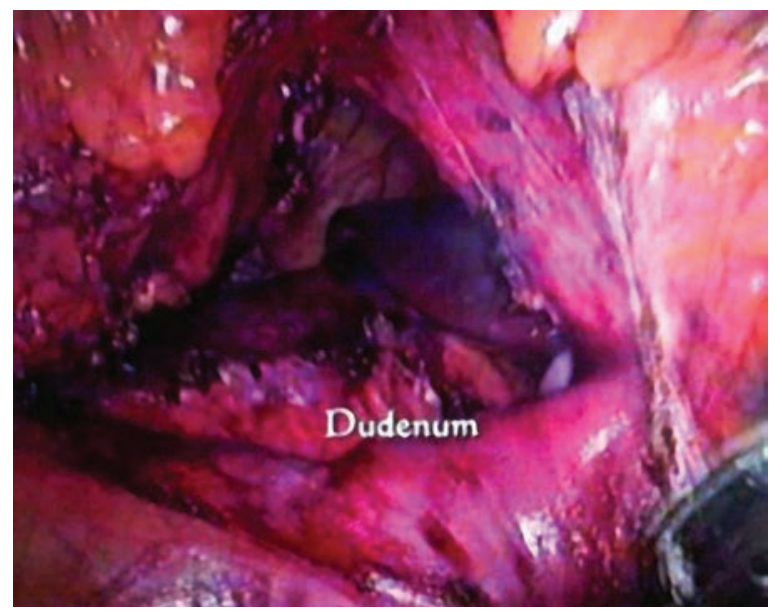

Figure (3): Dissection of hepatic flexure.

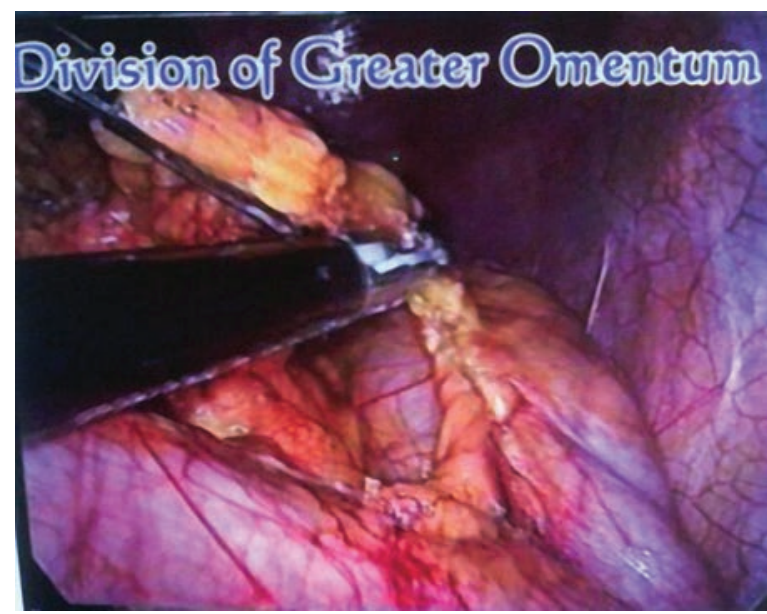

Figure (5): Division of greater omentum.

peritoneal cavity. In an approach from medial to lateral; the ileocolic, middle colic vessels, and the inferior mesenteric artery are divided. Medial mobilization is completed before the sigmoid is freed up laterally Figure (1,2). ${ }^{12}$

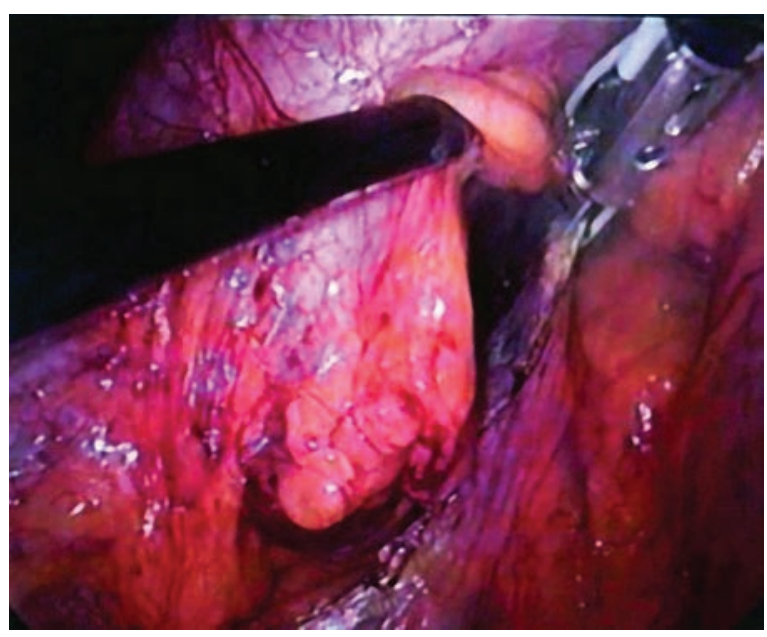

Figure (2): Dissection of right colon.

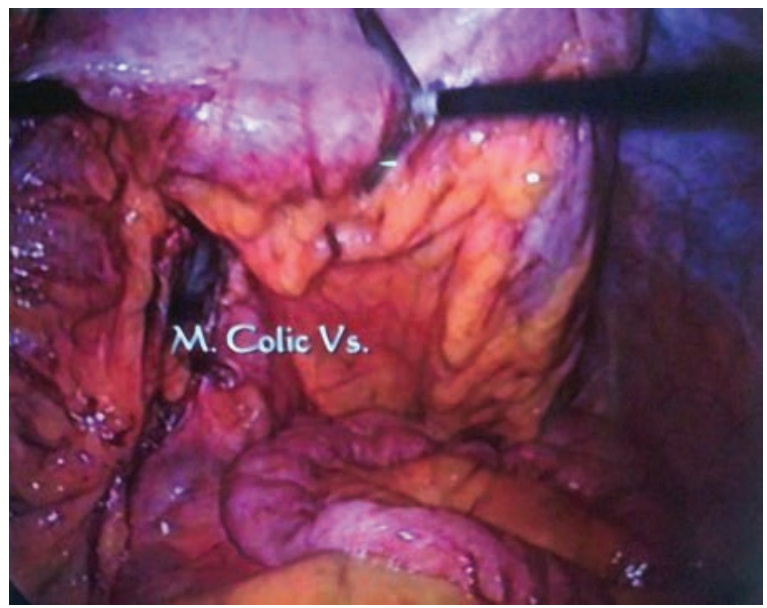

Figure (4): Division of middle colic Vs.

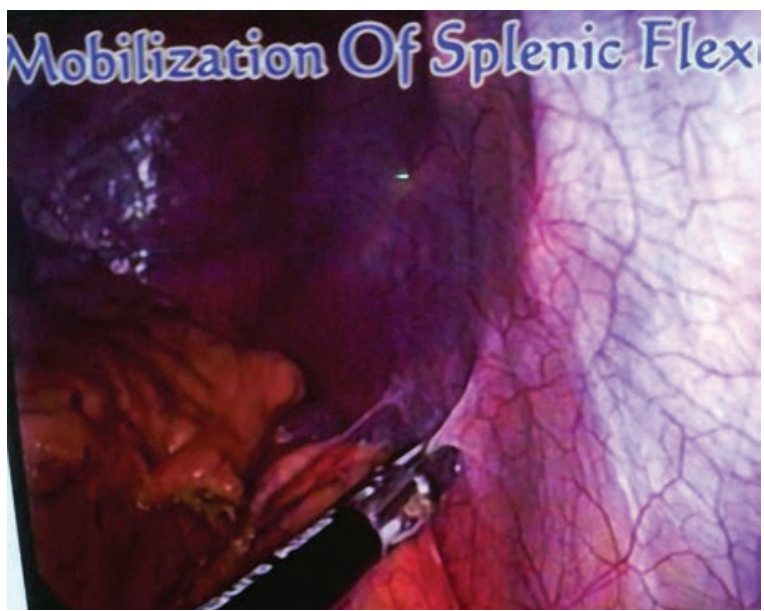

Figure (6): Mobilization of splenic flex.

The lateral mobilization of the descending, transverse, and ascending colon is done with the omentum being separated from the transverse colon completely Figure $(\mathbf{3}, \mathbf{4})$. The mesorectum is divided using the 


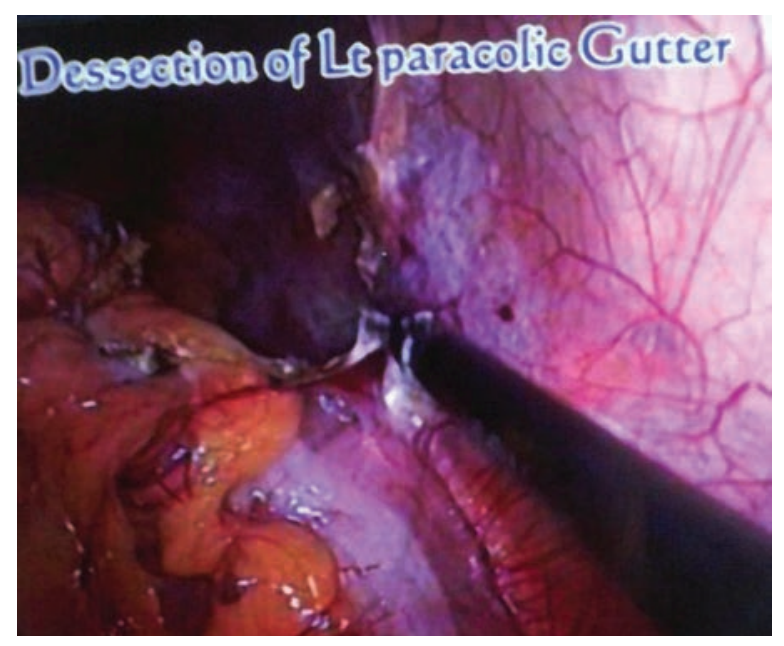

Figure (7): Dissection of Lt.paracolic gutter.

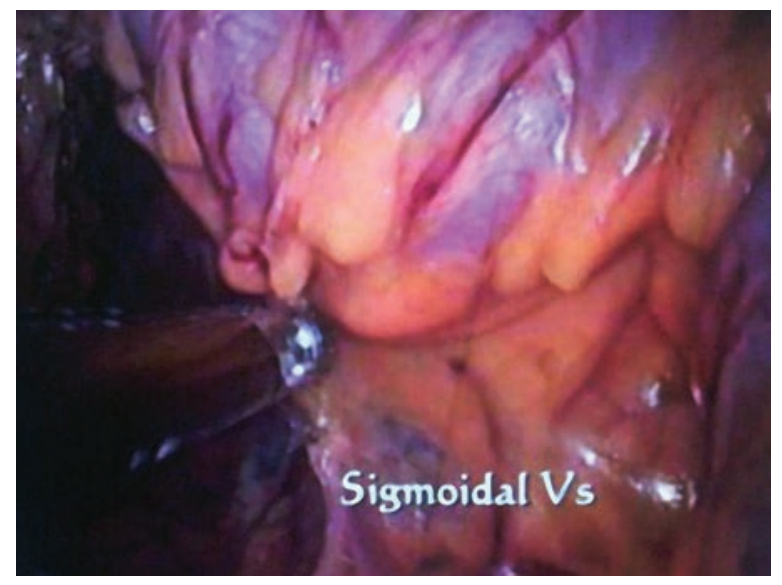

Figure (9): Dissection of sigmoid mesentry.

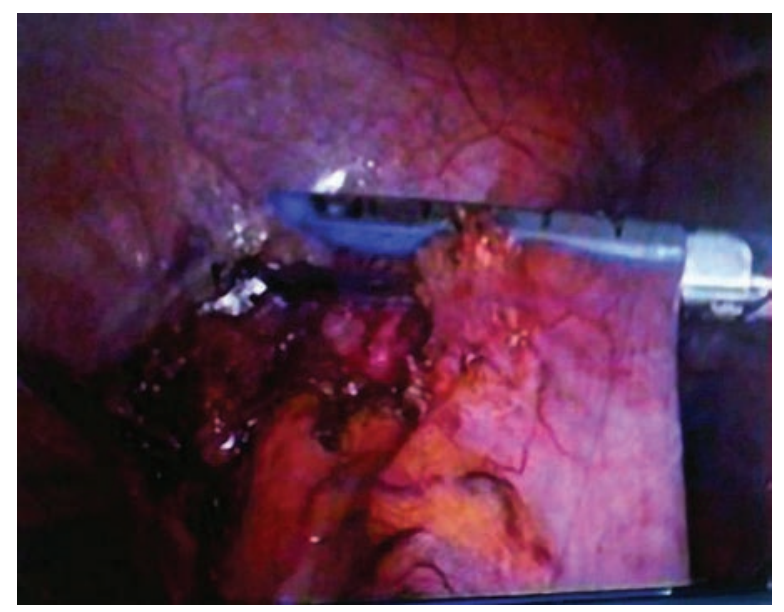

Figure (11): Transection of upper rectum.

harmonic scalped, and the upper rectum is transected with either one or two passes of the endoscopic linear cutting stabler Figure $(5,6,7)$. The colon is exteriorized through a 5-cm Pfennenstiel incision. The terminal ileum is transected extracorporeally. After pneumoperitoneum has been

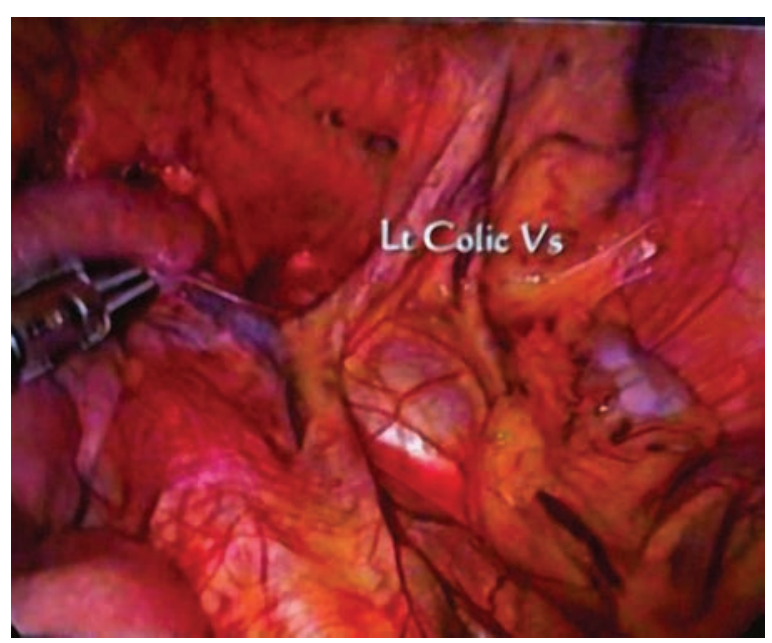

Figure (8): Division of Lt colic Vs.

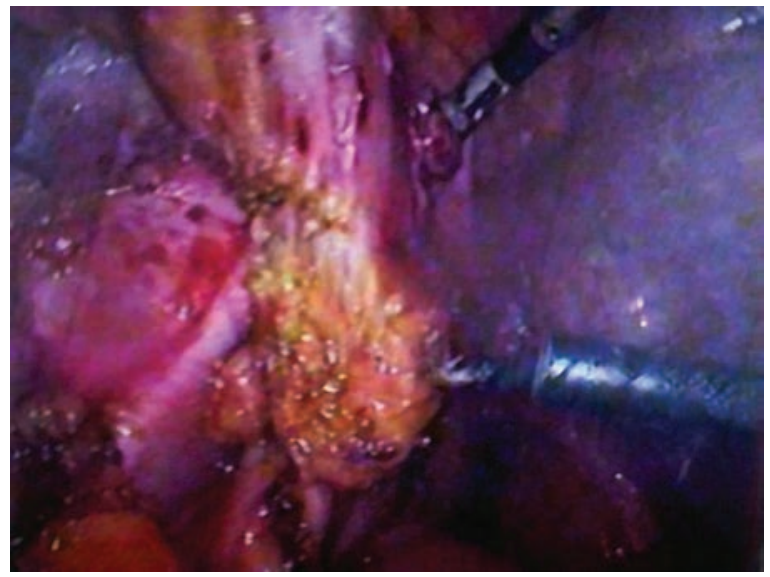

Figure (10): Dissection of mesorectum.

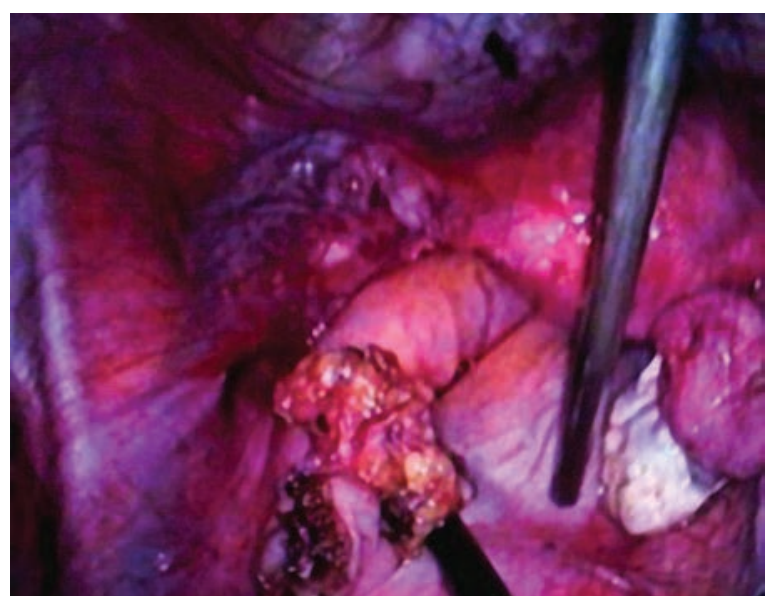

Figure (12): Rectal stump before IRA.

reestablished, the ileorectal anastomosis is performed laparoscopically using a doublestabling technique or end-to end anastomosis via circular stabler Figure (8,9,10,11,12). ${ }^{13,14}$

Perioperative management-data collection: All the patients underwent total laparoscopic colectomy with ileorectal anastomosis after 
Table 1: Epidemiological, and operative data of the patients.

$*($ mean \pm SD $)$

\begin{tabular}{|l|l|}
\hline Number of patients & 7 \\
\hline Age of the patients/year & $*(35 \pm 15.8)$ \\
\hline Sex, male : female & $1(14.2 \%): 6(85.7 \%)$ \\
\hline Operative data & \\
\hline Duration of procedure/min & $*(80 \pm 30 \mathrm{~min})$ \\
\hline Blood loss/ml & $*(75 \pm 30)$ \\
\hline Preoperative investigations & \\
\hline History, physical examination & $7(100 \%)$ \\
\hline Colonoscopy, barium enema & $7(100 \%)$ \\
\hline CT scan, MRI & $5(71.4 \%)$ \\
\hline Anorectal manometry & $6(85.7 \%)$ \\
\hline Colonic motility study & $7(100 \%)$ \\
\hline Colon transit-time testing & $7(100 \%)$ \\
\hline Defecography & $5(71.4 \%)$ \\
\hline EMG, PNTML & $4((57.1 \%)$ \\
\hline &
\end{tabular}

*EMG: Electromyography
* PNTML; Pudendal nerve terminal motor latency

Table 2 : Postoperative complication, and outcomes.

\begin{tabular}{|l|l|}
\hline Anastomotic leak & $1(14.2 \%)$ \\
\hline Intraperitoneal collection, abscess & $1(14.2 \%)$ \\
\hline Intraperitoneal hemorrhage & - \\
\hline Diarrhea, fecal incontinence & $3(42.8 \%)$ \\
\hline Small bowel obstruction & - \\
\hline Recurrent constipation & $2(28.4 \%)$ \\
\hline Median number of motions/day & $1(14.2 \%)$ \\
\hline $\begin{array}{l}\text { Median satisfaction, success rate, and } \\
\text { improved quality of life }\end{array}$ & 3 \\
\hline \% of reoperation & $82 \%$ \\
\hline Chest infection & - \\
\hline Pleural effusion & - \\
\hline Wound infection,hematoma & - \\
\hline GI bleeding & $1(14,2 \%)$ \\
\hline Overall morbidity\% & - \\
\hline Mortality\% & $38 \%$ \\
\hline Average hospital stay & - \\
\hline
\end{tabular}

*(mean \pm SD $)$

mechanical bowel preparation, and systemic antibiotic prophylaxis was administered. Oral feeding is regained 3days post-operatively, drains are removed with minimal serous fluid output. Evidence of anastomotic leak or intraperitoneal collections were assessed 
clinically and radiologically. Postoperative follow up was completed for all the patients, postoperative complications are demonstrated in Table (2).

\section{Results:}

In our study, 7 patients were presented with manifestations of slow-transit constipation, 6 were females $(85.7 \%)$, and 1 was male $(14.2 \%)$, average age of the patients was ( $35 \pm 15.8$ years), average operative time was ( $80 \pm 30 \mathrm{~min})$, average intraoperative blood loss was $(75 \pm 30 \mathrm{ml})$, average hospital stay was (6 \pm 2.5 days). No other procedures, or concurrent operations were performed. 3 patients (42.8\%) developed postoperative diarrhea and fecal incontinence which subsided completely after 2 weeks, 1 patient (14.2\%) had minor anastomotic leak and mild intraperitoneal collection which resolved on conservative measures. 1patient (14.2\%) had wound (Pfennenstiel incision) infection. No significant intraoperative, or postoperative hemorrhage was detected. The overall morbidity was $32 \%$, and no mortality could be detected. 2 patients (28.4\%) had recurrent abdominal pain and bloating which improved gradually over 2 months, 1 patient developed recurrent constipation which improved with cathertics, the median number of motions was 3/day, after about 3 weeks. After 3 months, a new colonic transit time study was performed and showed a regular transit. The median satisfaction, and success rate was $82 \%$ with marked improvement in quality of life which was detected via follow-up and clinical assessment. All the laparoscopic procedures have done after exclusion of defecatory disorders, and failure of conservative treatment. Malignancy or inflammatory bowel diseases were absent by histopathological examination for all resected specimens. There was no need for reoperation in all cases.

\section{Discussion:}

Chronic slow-transit constipation represents a disabling syndrome characterized by untreatable constipation, with a higher incidence in young female patients. The pathophysiology is still unclear, and a visceral neuropathy of the myoenteric plexus is supposed, together with abnormalities of neuroendocrine peptides. Less than $10 \%$ of the patientsrequire surgical management, in case of failure of conservative treatment and only after meticulous preoperative investigations. ${ }^{15}$

A total colectomy (TC) followed by ileorectal anastomosis (IRA) is the most common effective and widely performed surgical operation with a success rate of approximately $90 \%$, in spite of being associated with a significant postoperative morbidity, in terms of diarrhea, number of daily bowel motions, soiling, fecal incontinence, and postoperative adhesion. ${ }^{16}$

In 1995 (Lillehei and Wangesteen); first described the use of mechanical cecorectal anastomosis (CRA), after subtotal colectomy (SC). The original technique consists in an end to end CRA after a $180^{\circ}$ rotation of the cecum; after this, several modifications of such technique were described in order to avoid the possible vascular adhesions. The diffusion of laparoscopic surgery gave great impulse to this kind of operations. However, the debate regarding therapeutic choice is still open. ${ }^{17}$

Some authorssupport the SC because the preservation of terminal ileum, ileocecal valve, and cecum allows absorption of electrolytes, bile salts, and vitamin B12, and about 2 litres of water daily, preventing the ocurrance of renal, and biliary stones, with a reduced number of daily bowel motions, and without fecal incontinence. Furthermore, the cecum seems to fill well the pelvis, reducing the risk of postoperative obstruction, or adhesions of small bowel, compared to TC and IRA. Nevertheless, in some surgeons experience with the patients submitted to TC and IRA, a disabling diarrhea, incontinence or urgency was not observed at 6 months regular follow up. ${ }^{18,19}$

The functional results of SC and CRA were similar to those observed after TC and IRA with an excellent quality of life. Furthermore, aftera mean follow up of 43 months (range 23-73), a subocclusive crisis 
due to adhesions was never observed. In our study, 3 patients developed postoperative diarrhea, and fecal incontinence which resolved spontaneously after 2 weeks, 1 patient had minor anastomotic leak which also resolved with conservative measures, 2 patients developed abdominal pain and bloating, gradually improved without medical treatment. 1patient had recurrent constipation which is markedly improved on laxatives, the median number of motions 3/day, the median satisfaction, and success rate was $82 \%$, with marked improvement in quality of life. All such results are close to those published in the literature, and encouraging the usage of laparoscopy in treatment of chronic slowtransit constipation. ${ }^{20}$

\section{Conclusion:}

Laparoscopic TC and IRA represents efficacious, and interesting operation in the treatment of slow-transit constipation. Offering a good postoperative quality of life and a reduced morbidity compared to open surgery. Total laparoscopic operation allows reduced postoperative pain and ileus, a shorter hospitalization, a faster return to social activity along with a better cosmetic results, and good medium-long term functional results. ${ }^{21,22}$

\section{Reference:}

1- Agachan F, Chen F, Pfeifer J: A constipating scoring system to simplify evacuation and management of constipated patients. Dis Colon Rectum 1996; 39: 681-685.

2- Whitehead WE, Chaussade S, Corazziari E: Report of an international workshop on management of constipation. Gut 1999; 4: 99-113.

3- Martinelli E, Altomere DF, Rinaldi: Constipation after hysterectomy: Fact or fiction? Eur J Surg 2000; 166: 356-368.

4- Pfiefer J, Oliveira L, Park UC, Gonzales A: Are interpretations of video defecography reliable and reproducible? Int $J$ Colorectal Dis 1997; 12: 67-72.

5- Henry MM, Parke AG, Swash M: The pelvic floor musculature in the descending perineum syndrome. Br J Surg 2002; 69: 470-475.

6- Wexner SD, Daniel N, Jagelman DJ: Colectomy for constipation. Dis Colon
Rectum 2005; 34: 851-856.

7- Redmond JM, Smith GW, Barofsky A: Physiological tests to predict long-term outcomes of total abdominal colectomy for intractable constipation. Am J Gastroenterol 2004; 90: 784-753.

8- Barnes PR, Lennard-Jones JE, Hawley PR: Hirschsprung's disease and idiopathic megacolon in adults and adolescent. Gut 1998; 27: 534-514.

9- HoYH, GohHS:Theinvestigations of chronic constipation for surgical management. Sing Med J 1996 ; 37: 291-296.

10- Pemberton JH: Anorectal and pelvic floor disorders; putting physiology into practice. $J$ Gastroenterol Hepatol 2007; 115: 730-735.

11- Hinton JM, Lennnard-Jones JE, Young AC: A new method for studying gut transit time using radio opaque markers. Gut 1994; 121 : 842-847.

12- Piccirillo MF, Reisman P, Wexner SD: Colectomy as treatment for constipation in selected patients. Br J Surg 2003; 82: 889-891.

13- Yalcin S, Yalcin B, Gecim LE: Surgical intervention in patient with chronic constipation refractory to intensive medical treatment. Aus N Z J Surg 2007; 67: 562-565.

14- Wexner SD, Marchetti F, Salonga VD, et al: Neurophysiologic assessment of the anal sphincter. Dis Colon Rectum 2003; 34: 606-612.

15- Candelli M, Nista EC, Zocco MA, Gasbarini A: Idiopathic chronic constipation. Pathophysiology, diagnosis and treatment. Hepato Gastroenterology 2001; 48: 1050-1057.

16- Wald A: Constipation. Med Clin North Am 2006 ; 84: 1231-1246.

17- Bassotti G, Chiarioni G, Imbimbo B, et al: Impaired colonic response to cholinergic stimulation in patients with severe chronic idiopathic slow- transit constipation. Dig Dis Sci 1998; 38: 1040-1047.

18- Knowles CH, Scott SM, Lunniss PJ: Slowtransit constipation; a disorder of pelvic autonomic nerve? Dig Dis Sci 2004; 46: 389-401.

19- Nyam DC, Pemperton JH, Rath DM: Long term results of surgery for chronic constipation. Dis Colon Rectum 2007; 40: 273-279.

20- Lillehei RC, Wangensteen $\mathrm{OH}$ : Bowel function after colectomy for cancer, polyps, and diverticulitis. JAMA 2005; 59: 163-170. 
21- Hsiao KCW, Wu CC: Hand-assisted laparoscopic colectomy for slow transit constipation. Int J Colorectal Dis 2008; 23: 419-424.
22- Kessler H, Hohenberger W : Laparoscopic total colectomy for slow-transit constipation. Dis Colon Rectum 2005; 48: 860-861. 